to be a more robust way of testing efficacy and one not as vulnerable as the acute placebo-controlled studies to centre effects, or variable placebo response rates. It is for this reason that many of us turn to long-term treatment studies for proper reassurance about the efficacy of an antidepressant. I have no doubts from the results of the study that sertraline is indeed effective in long-term treatment.

Montgomery, S. A., Doogan D. P. \& Burnside R. (1991) The influence of different relapse criteria on the assessment of long term efficacy of sertraline. International Clinical Psychopharmacology, 6 (suppl. 2), 37-46.

Academic Department of Psychiatry

STUART MONTGOMERY

St Mary's Hospital

Praed St

London W2 INY

\section{Reports of the death of factor analysis are greatly exaggerated}

SIR: Bech et al (Journal, February 1992, 160, 206-211) presented psychometric analyses of the Hamilton scales for depression and for anxiety, and the SCL-90. The results, from a large and rigorous study, are fascinating and have major implications for cross-cultural psychiatric research. However, a subplot in the paper appears to be a "head-to-head" comparison of the merits of two psychometric methods: item-response analysis using the Rasch model versus exploratory factor analysis. This subplot is revealed in lines in the discussion: "Factor analysis is still considered to be an important psychometric method, but this study has demonstrated the difficulties in interpreting the results of such analysis." (pp. 209-210) and "The advantage of latent structure analysis has been demonstrated in this study." (p. 210). This last sentence continues a widely used misnomer as both Rasch model-item analysis and factor-analytic methods seek to reveal latent structure in item data: the former can reveal nonlinear item-response parameters on a single latent dimension of variation (e.g. depression, anxiety, general psychological distress); the latter can reveal multiple linear dimensions in data at the cost of assumptions about linearity of response that are not made in Rasch model-item analysis.

The presentation in the paper oversimplifies the situation: item analyses can use non-linear item responses to reveal the fit between the data and a model which assumes a single underlying latent dimension. Furthermore, these methods can test the homogeneity of the scaling in relation to apparent position on that dimension or in relation to other variables such as the counties in which the study took place. As all multiple-choice responses must be linear these are desirable abilities. However, current Rasch methods cannot reveal multidimensional latent variable structure. This is relevant in psychiatric scales such as the HRSD where there might be dimensions not only of severity of depression but also of the nature of the depressive symptoms-primarily somatic versus primarily psychological. Factor, for example, analytic methods can reveal multidimensional structure but only (with the exception of certain methods not used in this study) at the expense of the assumption of response linearity. These issues have been developed in increasingly mathematical terms by McDonald (1965, 1981, 1982; McDonald \& Ahlawat, 1974).

Unfortunately, Bech et al also skew the comparison between Rasch and factor-analytic methods by ignoring much psychometric research published in the last 20 years refining the use of factor-analytic methods: they apply the "eigenvalue greater than 1.0 " criterion to decide the number of factors to rotate despite the fact that this has been shown to be a very bad indicator of the best number of important factors (e.g. Zwick \& Velicer, 1986); they also use an arbitrary criterion to define the significance of factor loadings and apply no systematic indices of factor congruence.

This is not just an issue of parochial concern to psychometric hobbyists: multidimensional latent continuum structure is highly plausible in such data and the clarification of the extent to which such structure is, or is not, congruent across countries is an issue of very great importance as Bech et al note. A more extensive presentation of factor analytic results could considerably extend the insights Rasch analyses have given.

We would invite the authors to make their item data available to us to allow an analysis using factor-analytic methods. If, as we suspect, the factoranalytic methods do provide further insights into cross-cultural differences and consistencies we would submit a joint short communication to this Journal to complement the published excellent Rasch analyses and to redress some of the unfair polarisation against factor analysis in that publication.

MCDONALD, R. P. (1965) Difficulty factors and non-linear factor analysis. British Journal of Mathematical and Statistical Psychology, 18, 11-23.

- (1981) The dimensionality of tests and items. British Journal of Mathematical and Statistical Psychology, 34, 100-117.

- (1982) Linear versus nonlinear models in item response theory. Applied Psychological Measurement, 6, 379-396.

- \& Ahlawat, K. S. (1974) Difficulty factors in binary data. British Journal of Mathematical and Statistical Psychology, 27, 82-99. 
ZWICK, W. R. \& VELICER, W. F. (1986) Comparison of five rules for determining the number of components to retain. Psychological Bulletin, 99, 432-442.

Section of Forensic Psychiatry

Chris Evans

Section of Biostatistics BRIDGET DOLAN

St George's Hospital Medical School

Jenner Wing

Cranmer Terrace

London SWI7 ORE

\section{Maternal viral infection and schizophrenia}

SIR: Sham et al (Journal, April 1992, 160, 461-466) suggest that "maternal viral infection is an important cause of schizophrenia". They imply that the robust finding of a winter-birth excess among schizophrenics is due, in part at least, to a viral effect. They go on to state that this viral effect can help to explain the reported decline in the incidence of schizophrenia in countries in which there has been "an improvement in the living conditions in recent decades".

The explanatory hypothesis rests on the grounds that the viral infection of the mother adversely affects the developing brain of the foetus, predisposing it to later schizophrenia. This would be consistent with the neurodevelopmental theory of schizophrenia, but certain important factors remain unexplained.

Firstly, one of the hallmarks of neurodevelopmental schizophrenia is the early age of onset (Murray et al, 1992). In an extensive review of the literature, Bradbury \& Miller (1985) found no consistent schizophrenic subtype to be more prone to the seasonality effect. However, Takei et al (1992) among others, have reported winter birth to be associated with schizophrenia of later onset. Additionally, in an epidemiologically-based study, Castle et al (1992) reported that later-onset 'paranoid' patients showed a winter-birth effect, while early-onset 'neurodevelopmental' patients did not.

Secondly, it is clear that it is males rather than females who show a particular vulnerability to the severe, early-onset "neurodevelopmental" form of schizophrenia (Castle \& Murray, 1991). It is thus intriguing that, in an analysis of the "schizophrenogenic effect" of the 1957 influenza epidemic, O'Callaghan et al (1991) reported that such an effect was confined to females. Furthermore, in England and Wales, Der et al (1991) reported that the rates of schizophrenia for both sexes have declined, and that the sex-ratio has remained much the same over the years. In Ireland, Waddington \& Youssef (1992) found the decline to be greatest for females and later- onset cases. Should the decline indeed be due to improved living conditions and less maternal viral infection, surely such an effect would be more emphatic in males?

Bradbury, T. N. \& Miller, G. A. (1985) Season of birth in schizophrenia: a review of evidence, methodology, and aetiology. Psychological Bulletin, 98, 569-594.

CAstle, D. J. \& MurraY, R. M. (1991) The neurodevelopmental basis of sex differences in schizophrenia. Psychological Medicine, 21, 565-575.

-, Wessely, S., Sham, P., et al (1992) Early onset schizophrenia: a preponderance of males with severe illness associated with premorbid dysfunction. Schizophrenia Research, 6, 103-104.

DER, G., GUPTA, S. \& MURRAY, R. M. (1990) Is schizophrenia disappearing? Lancet, 335, 513-516.

Murray, R. M., O'Callaghan, E., Castue, D. J., et al (1992) A neurodevelopmental approach to the classification of schizophrenia. Schizophrenia Bulletin (in press).

O'Callaghan, E., Sham, P. C., TAKE, N., et al (1991) Schizophrenia after prenatal exposure to 1957 A2 influenza epidemic. Lancet, 337, 1248-1250.

Take, N., O'Callaghan, E., Sham, P., et al (1992) Winter birth excess in schizophrenia: its relationship to place of birth. Schizophrenia Research, 6, 102.

W ADDINGTON, J. L. \& Yousser, H. A. (1992) The declining incidence of schizophrenia in a rural Irish population of unusual homogeneity: secular trend towards early onset and male preponderance. Schizophrenia Research, 6, 101.

David Castle

Genetics Section

Michael Gill

Institute of Psychiatry

De Crespigny Park

London SE5 8 AF

AUTHOR'S REPLY: The concept of 'neurodevelopmental' schizophrenia was proposed on the basis that early onset and male cases of schizophrenia have a high frequency of obstetric complications, childhood personality and cognitive problems, and dystrophic brain abnormalities. By proposing this concept (Murray \& O'Callaghan, 1991), we hoped to promote a developmental perspective on schizophrenia, and yet draw attention to possible clinical and aetiological heterogeneity.

Drs Castle \& Gill surmise that if prenatal exposure to influenza epidemics increases the risk of subsequent schizophrenia in the unborn child by impairing neurodevelopment, then the season-ofbirth effect ought to be most obvious in early-onset and male cases of schizophrenia. They point out, however, that some reports suggest that the late winter/spring excess of births is most evident in lateronset and female (see Boyd et al, 1986) cases. It is also true that we regard 'neurodevelopmental' schizophrenia as having a poor prognosis, and yet two studies claim that the season-of-birth effect is greater 